\title{
Resolving intercontinental pollution plumes in global models of atmospheric transport
}

\section{Citation}

Rastigejev, Yevgenii, Rokjin Park, Michael P. Brenner, and Daniel J. Jacob. 2010. "Resolving Intercontinental Pollution Plumes in Global Models of Atmospheric Transport." Journal of Geophysical Research 115 (D2). doi:10.1029/2009jd012568.

\section{Published Version}

doi:10.1029/2009JD012568

\section{Permanent link}

http://nrs.harvard.edu/urn-3:HUL.InstRepos:14764380

\section{Terms of Use}

This article was downloaded from Harvard University's DASH repository, and is made available under the terms and conditions applicable to Other Posted Material, as set forth at http:// nrs.harvard.edu/urn-3:HUL.InstRepos:dash.current.terms-of-use\#LAA

\section{Share Your Story}

The Harvard community has made this article openly available.

Please share how this access benefits you. Submit a story.

\section{Accessibility}




\title{
Resolving intercontinental pollution plumes in global models of atmospheric transport
}

\author{
Yevgenii Rastigejev, ${ }^{1}$ Rokjin Park, ${ }^{2}$ Michael P. Brenner, ${ }^{3}$ and Daniel J. Jacob ${ }^{3}$ \\ Received 30 May 2009; revised 7 September 2009; accepted 17 September 2009; published 22 January 2010.
}

[1] Synoptic-scale pollution plumes in the free troposphere can preserve their identity as well-defined structures for a week or more while traveling around the globe. Eulerian chemical transport models (CTMs) have difficulty reproducing these layered structures due to numerical plume dissipation. We show that this dissipation is much faster than would be expected from the order of the advection scheme because of interaction between numerical diffusion and the nonuniformity of the atmospheric flow. The nonuniform flow stretches out the plume, enhancing the effect of numerical diffusion. For sufficiently strong stretching, the numerical decay of the plume is independent of the model grid resolution and is set instead by the flow Lyapunov exponent $\lambda$. In this regime, conventional numerical methods are not convergent: upon increasing grid resolution, the plume still decays with the same decay rate. The critical plume size below which the numerical scheme does not converge is set by the geometric mean of the grid spacing and the characteristic length scale $l=v / \lambda$ over which the flow varies, where $v$ is the wind speed. Above this critical plume size the numerically induced decay rate of the plume scales like the square root of the grid spacing. Application to an intercontinental pollution plume in a global CTM with realistic atmospheric flow shows that proper simulation of such a plume would require an impractical increase in grid resolution. Novel methods such as adaptive grids or embedded Lagrangian plumes are needed.

Citation: Rastigejev, Y., R. Park, M. P. Brenner, and D. J. Jacob (2010), Resolving intercontinental pollution plumes in global models of atmospheric transport, J. Geophys. Res., 115, D02302, doi:10.1029/2009JD012568.

\section{Introduction}

[2] Atmospheric pollutants originate from concentrated sources such as cities, power plants, and biomass fires. They are injected in the troposphere where eddies and convective motions of all sizes act to shear and dilute the pollution plumes as they are advected downwind. Despite this shear and dilution, observations from aircraft, sondes, and satellites show that pollution plumes in the remote free troposphere can preserve their identity as well-defined layers for a week or more as they are transported on intercontinental scales [Newell et al., 1999; Thouret et al., 2000; Colette and Ancellet, 2005]. This structure cannot be reproduced in the standard Eulerian chemical transport models (CTM) used for global modeling of tropospheric composition. The model plumes dissipate far too quickly [Staudt et al., 2003; Hudman et al., 2004; Fairlie et al., 2007; Vuolo et $a l ., 2009]$. This problem affects the ability of the models to properly represent the long-range transport patterns, radia-

\footnotetext{
${ }^{1}$ Department of Mathematics, North Carolina Agricultural and Technological State University, Greensboro, North Carolina, USA.

${ }^{2}$ School of Earth and Environmental Sciences, Seoul National University, Seoul, South Korea.

${ }^{3}$ School of Engineering and Applied Sciences, Harvard University, Cambridge, Massachusetts, USA.
}

Copyright 2010 by the American Geophysical Union. 0148-0227/10/2009JD012568\$09.00 tive forcing, nonlinear chemical evolution, aerosol dynamics, and surface impacts of the plumes. It compromises the use of the models for interpreting and assimilating satellite observations, obviously so for lidar instruments with high vertical resolution such as CALIOP [Winker et al., 2007] but also for passive instruments with altitude-dependent sensitivity.

[3] Modeling the transport of pollution plumes emitted from point sources is a classic problem in atmospheric chemistry, first addressed in urban and regional CTMs over 3 decades ago. A Lagrangian approach is obviously most appropriate to simulate isolated plumes but has difficulties in dealing with nonlinear chemistry and uniformity in the solution presentation (avoidance of particle clusters and voids). For these reasons, modelers have generally preferred an Eulerian approach for computational simulation of atmospheric chemical transport.

[4] A standard compromise for urban/regional modeling has been to embed Gaussian plumes within the Eulerian framework. The plumes evolve independently from the background air until they reach grid size, at which point they merge with the background air. The Gaussian plume is a standard parameterization of atmospheric turbulence based on analogy with molecular diffusion, i.e., assuming that turbulence is driven by eddies much smaller than the mean advection spatial scale (or, in an Eulerian model, than the model grid scale). Growth rates for Gaussian plumes can 
be specified as empirical functions of local atmospheric stability [Seinfeld and Pandis, 1998].

[5] A different but related problem emerged in the stratospheric modeling community about 2 decades ago with the need to represent filamented structures (also called ribbons or streamers) produced by wind shear in the strongly stratified stratospheric flow [Prather and Jaffe, 1990]. Resolving these structures is of particular importance for simulating transport at the edge of the polar vortices [Pierce et al., 1999; Waugh, 1993; Pierce and Fairlie, 1993] or mass exchange between the tropical pipe and midlatitudes [Weaver et al., 2000]. It has motivated the development of Lagrangian models of stratospheric transport, including various algorithms to address the above mentioned weaknesses of such models [Fairlie et al., 1999; McKenna et al., 2002]. The Lagrangian models describe the deformation and dissipation of the filaments on the basis of the flow divergence as measured by Lyapunov exponents [Prather and Jaffe, 1990; Legras et al., 2005]. Comparisons to observations show that the Lagrangian models are far more effective than their Eulerian counterparts in generating and preserving the filamentary structures [Khosrawi et al., 2005], although this capability can be improved in Eulerian models by using an isentropic vertical coordinate [Weaver et al., 2000; Reames and Zapotocny, 1999].

[6] Interest in pollution plumes transported in the troposphere on intercontinental scales is more recent and presents yet another type of modeling problem. Instead of diluting over a timescale $\sim 1$ day in the planetary boundary layer (PBL), as in the Gaussian plumes of urban/regional models, these plumes are lifted to the free troposphere where they can maintain their structure for a week or more. Instead of being dissipated by small-scale eddies, they are stretched and dissipated by large-scale differential motions (wind shear, chaotic advection), eventually reaching sufficiently small thicknesses that small-scale eddy diffusion can complete the mixing. These considerations would make them more similar to stratospheric filaments. However, the tropospheric flow is far more variable than the stratospheric flow, so that the dispersion and dissipation of tropospheric plumes may involve different controlling processes than for the stratospheric filaments. The lifetimes of plumes are also much shorter in the troposphere than in the stratosphere, rarely extending beyond 1 week, so that issues such as noise in the meteorological assimilation fields are less important [Stohl et al., 2004].

[7] It is in fact not immediately clear why global Eulerian CTMs would have such difficulties in simulating intercontinental pollution plumes, considering that the typical dimensions of these plumes $(\sim 1000 \mathrm{~km}$ in the horizontal, $\sim 1 \mathrm{~km}$ in the vertical) are commensurate with the typical grid resolution of the models. It might seem that a modest increase in grid resolution, combined with an accurate advection scheme, would solve the problem. The goal of the present paper is to explain why this is not so. Modern advection codes use high order numerical schemes with minimal numerical diffusion [Colella and Woodward, 1984; Lin and Rood, 1996]. For highly resolved situations, high order schemes lead to rapid convergence to the correct solution with grid refinement. However, at the relatively coarse resolutions required for global CTMs (with $\sim 100 \mathrm{~km}$ horizontal mesh spacing), we demonstrate that the effect of numerical diffusion, interacting with the complex velocity field of the atmospheric flow, leads to a greatly enhanced numerical decay rate of the plume. This is due to the fact that the dilution rate of a plume advecting in a complex velocity field is greatly enhanced by even a small amount of (numerical) diffusion. We argue that in a typical atmospheric flow, the high order accuracy of the underlying numerical schemes could only be recovered with an impractical increase in grid resolution.

[8] The next section illustrates the plume dissipation problem with simulations using the GEOS-Chem global CTM [Bey et al., 2001] as a prototype. Section 3 describes the theory for the plume dilution due to numerical diffusion. To verify and illustrate the developed theory, section 4 shows simulations of a tracer plume propagating in an ideal convergent-divergent flow, followed by a tracer plume in a real atmospheric flow.

\section{Dissipation of Pollution Plumes}

[9] Atmospheric CTMs describe the three-dimensional (3-D) concentration fields of chemicals under the influence of atmospheric transport and chemical reactions. The equations of motion, in Eulerian form, solve for the evolution of $C_{i}(\mathbf{x}, t)$, the mass fraction (below referred as "concentration") of the $i$ th chemical species

$$
\frac{\partial C_{i}}{\partial t}+\mathbf{u} \cdot \nabla C_{i}=R_{i}+s_{i}(\mathbf{x}, t) \quad i=1 \ldots N
$$

Here $\mathbf{u}$ is the wind velocity, $R_{i}$ is the net chemical production rate (typically a function of the mass fractions of other chemicals), and $s_{i}$ describes local emissions and nonchemical sinks. Molecular diffusion is negligible on the scales of interest. The wind velocity is typically provided by an assimilated meteorological data base on a (latitude, longitude) grid. Depending on the problem addressed, the number of interacting chemical species and chemical reactions may be large, placing computational limits on the grid resolution that can be achieved.

[10] In what follows, we consider the transport of a plume in the free troposphere in the absence of convection (which would cause rapid vertical mixing and dissipation of the plume). Under these conditions, the flow is stratified vertically and plume dissipation is expected to take place primarily as a result of the horizontal flow.

[11] To illustrate the nature of the problem, we first conducted a prototypical simulation of a global CTM, in which an inert tracer was released instantaneously in the free troposphere over China in a 2-D (horizontal) implementation of GEOS-Chem. All calculations described in this paper are performed with the PPM scheme. The initial plume has a size $10^{\circ} \times 15^{\circ}$ degrees, resolved by 5 and 6 grid squares in longitudinal and latitudinal directions, respectively. We first assume that the flow is spatially and temporally uniform. The blue line in Figure 1 shows the concentration in the plume as a function of time. Without any dissipation or dispersion, the concentration $C_{i}$ remains constant in time, with a parcel of fluid originating at $\mathbf{x}_{0}$ following the trajectory

$$
\frac{d \mathbf{x}}{d t}=\mathbf{u}(\mathbf{x}, t),
$$




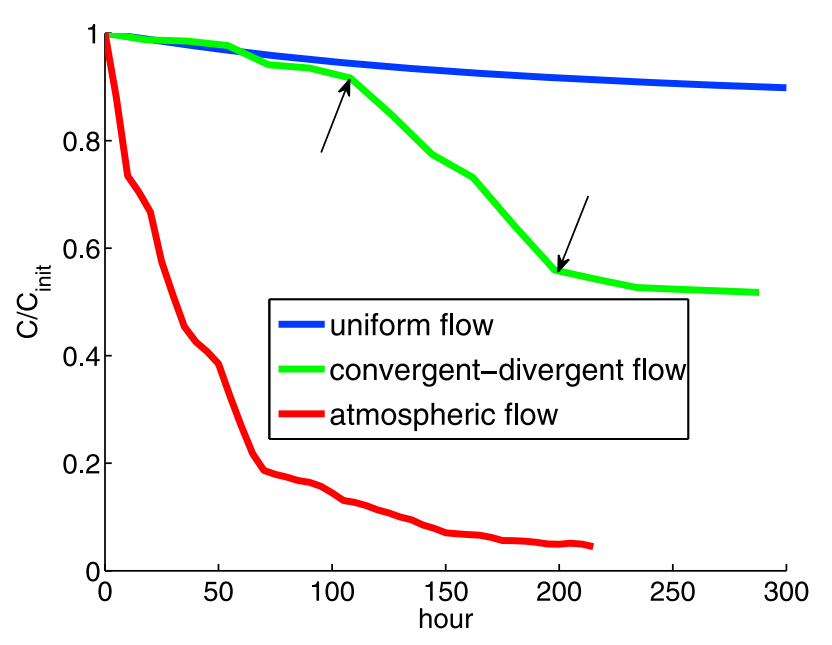

Figure 1. Maximum concentration of a plume as a function of time, as calculated by GEOS-Chem. An initially localized plume begins in China and propagates across the Pacific Ocean. To focus on the role of flow enhanced numerical dissipation, both chemical reactions and convection have been turned off. The blue line represents the maximum plume concentration in a spatially uniform flow; the red line corresponds to the atmospheric flow shown in Figure 2. The green line represents a convergent-divergent flow, in which the Lyapunov exponent $\lambda$ is significant between times $125 \mathrm{~h} \leq t \leq 200 \mathrm{~h}$, as described in the text. The arrows denote the times at which the stretching turns on and off.

Instead, there is a slight decay in the maximum concentration, of order $10 \%$ over 10 days, resulting from the numerical dissipation. Such a small decay is generally acceptable for practical applications.

[12] Now we examine plume propagation in an actual horizontal flow field over the Pacific Ocean, shown in (a) time $=000$ hours

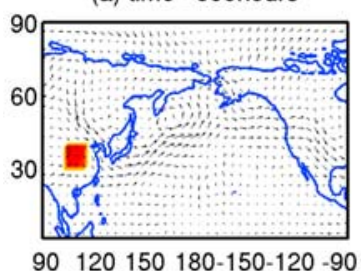

(c) time $=150$ hours

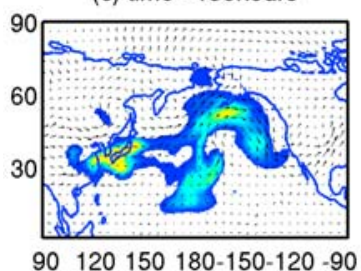

(b) time $=060$ hours

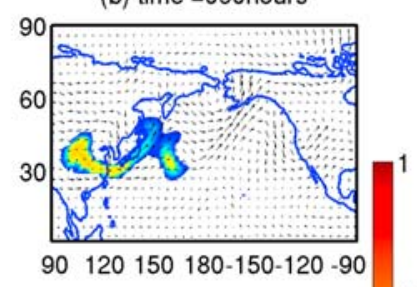

(d) time $=210$ hours

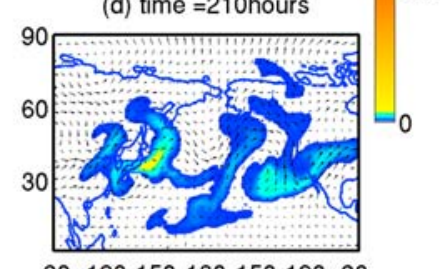

$90120150 \quad 180-150-120-90$
Figure 2. Evolution of a plume simulated in the free troposphere at $4 \mathrm{~km}$ altitude using GEOS-Chem in a twodimensional representative flow over the Pacific Ocean. The maximum concentration is given in the red line in Figure 1. Shown are times (a) $t=0$, (b) $t=60 \mathrm{~h}$, (c) $t=150 \mathrm{~h}$, and (d) $t=210 \mathrm{~h}$, respectively.
Figure 2. The plume is placed at $4 \mathrm{~km}$ over the land surface and begins its travel on 1 May 2001. The meteorological data is updated every $6 \mathrm{~h}$.

[13] Again, we turn off convection in the simulation to ensure there are no vertical flow fields influencing the propagation of the plume. To extract a 2-D velocity field from the three dimensional flow, we take the horizontal velocity field at a vertical layer $\approx 4 \mathrm{~km}$ off the ground. We then impose this velocity field at each vertical layer, so that the flow field is entirely two dimensional. We allow the velocity field to be time dependent, by modifying the horizontal flow field globally to mirror the change in our vertical slice.

[14] The situation here is dramatically different than in the case of a uniform flow. The plume is rapidly distorted and dissipated by the advection flow field. The red line in Figure 1 shows the maximum concentration in the plume as a function of time: the concentration strongly decays in time. The peak concentration in the plume decreases to $5 \%$ of the original value after 10 days, in sharp contrast to the uniform flow situation.

[15] The decay in the concentration field is quite dramatic; to demonstrate that this was not an artifact of our 2-D implementation, we carried out a number of tests, as shown in Figure 3. The red line shows the concentration decay for a simulation with the identical velocity field as Figure 1, but using the full two-dimensional number column density, as opposed to the maximum concentration. The blue line shows the decay of the plume for the fully three-dimensional flow. The decay rates of all of these cases are nearly identical, with the fully $3-\mathrm{D}$ plume showing slightly stronger decay.

[16] As a final check that the cause of the strong decay is indeed caused by the combination of stretching by the flow and numerical diffusion, we repeat the simulations with a

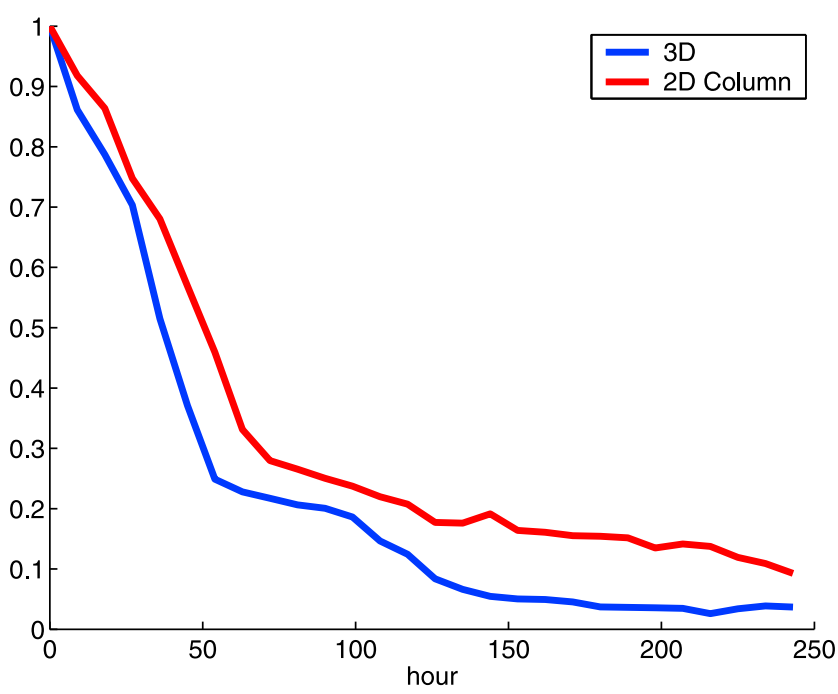

Figure 3. Maximum concentration of the plume as a function of time, as in the atmospheric flow of Figure 1, for several different representations of the atmospheric velocity flow. Whereas Figure 1 shows the maximum concentration in a two-dimensional flow, the red line in Figure 3 shows the full number density column. The blue line represents the maximum plume concentration for the fully threedimensional calculation. 
simpler flow, with a velocity gradient that is maintained over only part of the flow. We send the plume through a flow that is initially uniform, but then let it enter a region of stretching before reentering a uniform flow. The flow field used in this experiment is similar to that used in section 4.1, using equations (20) and (21) with $\epsilon=4 / 5$. The green line in Figure 1 show the decay of the plume; the decay rate of the plume transiently increases when it enters the region with stretching.

[17] It is worth noting that the plume decay observed here is stronger than that observed by Reames and Zapotocny [1999]. These authors study a gaussian plume which is quite well resolved in the horizontal direction (with the plume boundary being resolved by $12-13$ grid points (their Figure $2 \mathrm{c}$ ), which is much higher than $2-3$ grid points here). In the vertical direction, their resolution is much smaller, corresponding to 2-3 grid points. Therefore whereas the numerical diffusion in the current study is dominant in the horizontal, in the work of Reames and Zapotocny [1999] the diffusion dominates the vertical motion.

\section{Rapid Plume Decay in a Variable Flow}

[18] The simulations demonstrate that the numerical dissipation of the plume is strongly enhanced by stretching due to complex atmospheric flow fields. The question now is to determine which features of the flow fields are the root cause of the enhanced dissipation in order to determine a possible cure.

\subsection{Numerical Diffusion: A Simple Example}

[19] The obvious culprit is numerical diffusion. Numerical solutions to advection equations necessarily contain artifacts which cause numerical diffusion or dispersion, well discussed in both the numerical analysis [LeVeque, 1990; Hoffman, 1992] and atmospheric chemistry literatures [Lin and Rood, 1996]. The size of these artifacts, as well as whether they cause diffusion or dispersion, depends on the advection scheme chosen, as well as the spatial resolution. What is particularly curious about the simulations here is that global CTMs like GEOS-Chem use high order advection schemes which are designed to minimize these numerical effects.

[20] Nonetheless, we contend that numerical diffusion is the root cause of the strong decay. To explain this, it is first helpful to review the reasons for numerical diffusion or dispersion. We begin by considering a simple (unsophisticated) numerical method for solving the advection equation

$$
\frac{\partial C}{\partial t}+v \frac{\partial C}{\partial x}=0
$$

We represent $C(x, t)$ as values on a discrete mesh $x_{i}=i \Delta x$ at times $t=t_{m}=m \Delta t$, so that $C_{i}^{m}=C\left(x_{i}, t_{m}\right)$. One of the simplest numeral schemes for solving this problem is then given by

$$
\frac{C_{i}^{m+1}-C_{i}^{m}}{\Delta t}+v \frac{C_{i}^{m}-C_{i-1}^{m}}{\Delta x}=0 .
$$

Given the solution at a given time $t_{m}$, we can use this equation to obtain the solution at a time $t_{m+1}$. We can evaluate the error in the numerically computed solution by studying how the numerical scheme equation (4) reduces to the original equation when $\Delta x, \Delta t \rightarrow 0$. To do this, we Taylor expand $C_{i}^{m+1}=C(x, t+\Delta t)=C(x, t)+\Delta t \partial_{t} C(x, t)+$ $\left(\Delta t^{2} / 2\right) \partial_{t}^{2} C+\ldots$ and $C_{i-1}^{m}=C(x-\Delta x, t)=C(x, t)-$ $\Delta x \partial_{x} C(x, t)+\left(\Delta x^{2} / 2\right) \partial_{x x} C+\ldots$, and then insert these into equation (4). After some algebra we obtain

$$
\frac{\partial C}{\partial t}+v \frac{\partial C}{\partial x}=D \frac{\partial^{2} C}{\partial x^{2}}+\ldots
$$

where

$$
D=\frac{\Delta x^{2}}{2 \Delta t}\left(1-\sigma^{2}\right)
$$

and $\sigma=v \Delta t / \Delta x$ is the Courant number.

[21] Equation (5) shows that the largest error in the numerical scheme is caused by the $D \partial_{x x} c$ term; If we fix the ratio of $\Delta x / \Delta t$, then as $\Delta x \rightarrow 0$, this numerical error vanishes, proving that the numerical scheme converges to the desired equation. (This is required for numerical stability; note that if $\sigma>1$ the diffusion constant is negative and the method is therefore unstable.) However at any finite $\Delta x$, $\Delta t$ the effect of the numerical error is precisely analogous to physical diffusion. This numerical scheme is said to be first order, since the error vanishes linearly in the mesh spacing $\Delta x$.

[22] The effect of numerical diffusion can be mitigated by using a more sophisticated advection scheme. For example, GEOS-Chem uses the TPCORE algorithm [Lin and Rood, 1996] for tracer advection. TPCORE is a multidimensional flux-form conservative upstream-based transport scheme. The algorithm uses the Piecewise Parabolic Method (PPM) [Colella and Woodward, 1984] for Courant numbers smaller than unity and a semi-Lagrangian approach for Courant numbers larger than unity. The latter is mainly for polar regions where gridboxes shrink to small sizes.

[23] For smooth solutions, when the magnitude of $c(x, t)$ varies slowly on the length scale of the mesh spacing, such higher order methods lead to corrections that are second or third order accurate in the mesh spacing, depending on the scheme used (e.g., second order for the Van Leer method and third order for PPM). For higher order equations, the numerical effects are qualitatively different. For example, if a scheme were second order accurate, the analogue of equation (5) would be

$$
\frac{\partial C}{\partial t}+v \frac{\partial C}{\partial x}=\kappa \frac{\partial^{3} C}{\partial x^{3}}+\ldots
$$

where $\kappa \sim(\Delta x)^{3} / \Delta t$. The numerical error introduces dispersion into the solution.

[24] During the model transport of a pollution plume, it is often the case that the size of the plume is of order of only a few mesh boxes; there are in that case large gradients from one mesh box to the next. Whenever such large gradients exist, the numerical method will be first order accurate, and the dominant numerical effect will be diffusion. Indeed, in Appendix A we analyze the van Leer method with a similar method outlined here and demonstrate that the numerical 
error for such discontinuous solutions again corresponds to first order diffusion, with $D$ scaling linear with the mesh spacing $\Delta x$

$$
D=\eta v \Delta x
$$

where $v$ is the advection speed and $\eta$ is a method-dependent constant. For example for the Van Leer method, $\eta=(1-\sigma) / 4$ [Van Leer, 1974].

\subsection{Numerical Diffusion and Plume Decay}

[25] The numerical solutions for pollution plumes whose extent is of order the mesh spacing therefore corresponds to the dynamics of a plume under the modified equation

$$
\frac{\partial C}{\partial t}+\mathbf{u} \cdot \nabla C=D \nabla^{2} C
$$

where $D$ is the numerical diffusivity. As emphasized above, in the absence of numerical diffusion $(D=0)$, a plume with initially constant concentration will maintain constant concentration for all time; with nonzero $D$, the concentration will decay with time. Naively, one would expect that the numerically induced decay rate of the plume will scale linearly with $D$ and hence approach zero linearly as $\Delta x \rightarrow 0$. Indeed, the order of convergence of a numerical scheme is defined as the rate at which the numerical error decreases as $\Delta x \rightarrow 0$; a scheme is first order if the error vanishes linearly in $\Delta x$, second order if it vanishes quadratically $\left(\Delta x^{2}\right)$, etc. We will see below that the physics of advection-diffusion can lead to reduction of the order of convergence of the numerical scheme and hence to much higher numerically induced decay rates, especially when the numerical solution is not highly resolved.

[26] To determine the rate at which plumes diffuse, we now consider how the decay rate of the plume depends on both $D$ and the velocity field $\mathbf{u}$. For typical atmospheric simulations, the transport due to numerical diffusion is much smaller than the transport by the flow. A plume in a complex velocity follows the streamlines of the flow, with the shape of the tracer plume being stretched by the flow field. If we assume for conceptual simplicity that the tracer concentration is uniform in the plume, then the rate of decay of $C$ is given by the diffusive outflux through the boundary, namely

$$
V \frac{d C}{d t}=D S \mathbf{n} \cdot \nabla C
$$

where $\mathbf{n}$ is the direction normal to the plume, $V$ is the volume of the plume, and $S$ is the surface area of the plume. Now, let us define the characteristic length scale $r_{\mathrm{b}}$ over which the tracer concentration decays from the concentration $C$ inside the plume to zero outside the plume. With this length scale defined, we can express the decay rate of a plume as

$$
V \frac{d C}{d t}=-D S \frac{C}{r_{\mathrm{b}}},
$$

or setting $V / S=W$, where $W$ is the width of the plume, we have

$$
\frac{d C}{d t}=-D \frac{C}{r_{\mathrm{b}} W}
$$

(In reality, $\mathrm{V} / \mathrm{S}=\mathrm{kW}$, where $k$ is a shape-dependent prefactor of order unity. Here, we assume that $k=1$.) Equation (12) then implies that the concentration in the plume decays exponentially, $C \sim e^{-\alpha t}$, with the decay rate

$$
\alpha=\frac{D}{r_{\mathrm{b}} W} .
$$

[27] On the surface, equation (13) suggests that the concentration in the plume decays exponentially, with the decay rate proportional to the numerical diffusivity $D$. However, this argument is fallacious, since we still have not yet determined what sets the characteristic length scale $r_{\mathrm{b}}$. Indeed, we will now show that depending on the circumstance, $r_{\mathrm{b}}$ can itself depend on the numerical diffusivity $D$; when this happens the decay rate of the plume is greatly enhanced over the naive estimate.

[28] What sets the length scale $r_{\mathrm{b}}$, over which the concentration in the plume decays from $C$ to zero? This length scale is determined by a balance between diffusion and the stretching of the plume along the streamlines of the flow. Intuitively, if $r_{\mathrm{b}}$ is small, diffusion will dominate and the plume boundary $\left(r_{\mathrm{b}}\right)$ will thicken; on the other hand, if the plume edge scale $r_{\mathrm{b}}$ is big, stretching dominates and the plume boundary $\left(r_{\mathrm{b}}\right)$ will shrink. In between these two extremes, there is an equilibrium plume thickness $r_{\mathrm{b}}$ for which diffusion and stretching are in balance.

[29] The stretching of the flow field is quantified by the Lyapunov exponent $\lambda$, defined as the exponential rate at which nearby trajectories diverge from each other [Balkovsky and Fouxon, 1999]; in a convergent flow

$$
\lambda=-\frac{\partial v}{\partial y}=\frac{\partial u}{\partial x},
$$

where $v(u)$ is the $y(x)$ component of the velocity.

[30] We can now translate the physical argument into mathematical terms: the diffusive broadening of the front happens with a rate $D / r_{\mathrm{b}}^{2}$; the stretching of the plume happens with a rate $\lambda$. Diffusion balances stretching when these two rates perfectly balance, namely

$$
\frac{D}{r_{\mathrm{b}}^{2}}=\lambda \text {. }
$$

This implies that $r_{\mathrm{b}}=\sqrt{D / \lambda}$. Note that if $r_{\mathrm{b}}$ is smaller than $\sqrt{D / \lambda}$ diffusion beats stretching, causing the front to broaden, whereas in the opposite limit stretching beats diffusion so the front sharpens. Using this result for $r_{\mathrm{b}}$ in equation (13) implies that

$$
\alpha=\frac{\sqrt{D \lambda}}{W}=\frac{\sqrt{\eta v \Delta x \lambda}}{W} .
$$

[31] Note that equation (16) implies that the decay rate $\alpha$ of the plume increases with decreasing width $W$. But the width of a plume does not remain constant: as the plume stretches, $W$ will become smaller and smaller. The width cannot decrease indefinitely: the smallest the plume width can become is $W=r_{\mathrm{b}}$. If the plume width were to decrease 
below $r_{\mathrm{b}}$, then diffusion would dominate stretching, causing the plume to thicken.

[32] What is the stretching rate of the plume when it is fully stretched, with $W=r_{\mathrm{b}}$ ? Equation (16) implies that the decay rate of the plume

$$
\alpha=\sqrt{D \lambda} / \sqrt{D / \lambda}=\lambda,
$$

namely the decay rate is equal to the Lyapunov exponent, and is independent of the numerical diffusivity D.

[33] This is a remarkable fact [Chella and Ottino, 1984; Balkovsky and Fouxon, 1999; Chertkov and Lebedev, 2003] and has an important consequence for the numerical scheme, as we will see below: once the plume is fully stretched, i.e., $W=r_{\mathrm{b}}$, the argument implies that the numerical scheme is not convergent. Initial decreasing of the mesh spacing will not change the decay rate of the plume until the mesh is refined enough to decrease $r_{\mathrm{b}}$ $\left(r_{\mathrm{b}}\right.$ changes as a square root of $\Delta x$, i.e., $r_{\mathrm{b}} \sim \sqrt{\Delta x}$ ), so that $W>r_{\mathrm{b}}$. As is shown below, further mesh refinement will cause slow (below first order) convergence of the numerical scheme.

[34] At what $r_{b}$ does this transition to $\alpha=\lambda$ occur? Since the numerical diffusivity $D=\eta v h$, we can evaluate

$$
r_{\mathrm{b}}=\sqrt{\frac{D}{\lambda}}=\sqrt{\frac{\eta v \Delta x}{\lambda}} .
$$

Estimating $\lambda \approx \nabla \mathbf{u} \sim U / \ell$, where $\ell$ is the characteristic length scale over which the flow is varying, and $U$ is the characteristic wind speed, we see that

$$
r_{\mathrm{b}} \approx \sqrt{\eta} \sqrt{\ell \Delta x} .
$$

The length scale $r_{\mathrm{b}}$ scales with the geometric mean of the mesh spacing and the length scale over which the velocity field varies. Note that if the flow field is well resolved, then $\ell \gg \Delta x$, so that $r_{\mathrm{b}}>\Delta x$.

[35] Note that this derivation implicitly assumes that the stretching dominates the flow. In the opposite limit where $D$ is very large, or the Lyapunov exponent $\lambda$ is small, the length scale $r_{\mathrm{b}}$ always remains much larger than the plume width. In either case the plume will decay according to normal diffusive dynamics. But the limit where stretching dominates diffusion is precisely the relevant one for analyzing numerical schemes relevant in atmospheric dynamics, since numerical diffusivities are designed to be very small.

\subsection{Dependence of Plume Decay Rate on the Mesh Spacing}

[36] We can now consider how mesh refinement influences the decay rate of the plume. If the plume width approaches $r_{\mathrm{b}}$ the decay rate $\alpha \sim \lambda$, so mesh refinement has no effect on plume decay. For plumes with width $W>r_{\mathrm{b}}$ the decay rate decreases like $\sqrt{\Delta x}$ according to (16) and also correlates with the Lyaponuv exponent $\lambda$. When $r_{\mathrm{b}}>W$, the plume dissipates according to standard diffusive broadening. If the plume is well resolved, this dissipation rate is determined by the order of the numerical method. However, for a plume with sharp boundaries of order the mesh spacing, the numerical scheme is effectively first order, so the decay rate is set by the numerical diffusivity and thus decreases approximately as a first order of the spatial refinement.

[37] A numerical scheme is said to converge if it approaches the desired behavior upon mesh refinement. This means that the decay rate $\alpha \rightarrow 0$ as $\Delta x \rightarrow 0$. In the first case when $W \sim r_{\mathrm{b}}$ a decrease in mesh spacing has no effect on the decay rate. In the second case $\alpha \sim \sqrt{\Delta x}$, which vanishes much more slowly compared with the linear decay of $\alpha$ expected from $D \sim \Delta x$.

[38] The poor convergence described herein could be exacerbated by the resolution effect discussed in a recent paper of Wild and Prather [2006] where it was shown that better resolution also improves the resolution of the velocity gradients and hence leads to increased stretching of the flow.

\subsection{A Consistency Check}

[39] There is one important consistency check on our analysis that is worth pointing out explicitly. Our entire derivation assumed that the plume was governed by the advection diffusion equation (9) and not by a higher-order equation (7). However, as emphasized there, the diffusion equation only describes the numerical error when the boundary of the plume is sharp; for smoothly varying solutions, the TPCORE algorithm used in GEOS-Chem is higher order, and equation (9) would not apply.

[40] According to our arguments above, the plume eventually will be smoothed out on the length scale $r_{\mathrm{b}}$. Using equation (19), this corresponds roughly to $r_{\mathrm{b}} / \Delta x \sim \sqrt{\ell / \Delta x}$ mesh boxes, where $\ell$ is the characteristic length scale of the flow. If we take $\ell=1000 \mathrm{~km}$ as the typical scale for horizontal flow variation over the Pacific Ocean, and $\Delta x=100 \mathrm{~km}$ as a typical mesh spacing, then $r_{\mathrm{b}}$ corresponds to about three grid boxes. Thus typical plumes will be quite underresolved, implying that our analysis is self consistent, in that the numerical error for propagating pollution plumes will be described by the diffusion equation. To resolve $r_{\mathrm{b}}$ by 10 mesh points (and hence be able to utilize the higherorder nature of the advection scheme) requires an approximately 10 -fold reduction in the mesh spacing $\Delta x$.

\section{Numerical Tests}

[41] We now carry out a set of numerical experiments to test the dependence of the decay rate on mesh spacing in the manner described above. To do this, we need to test the variation of the decay rate $\alpha$ with both Lyapunov exponent $\lambda$ and mesh spacing $\Delta x$. In the first part of this section we consider pollution plume propagation in a model convergent-divergent flow, while in the second part we consider the plume dynamics in a real atmospheric flow.

\subsection{Convergent-Divergent Flow}

[42] Here we construct an incompressible convergentdivergent flow in two dimensions, as a testing ground for how the plume decay depends on the structure of the flow. We need a flow in which we can systematically control the Lyapunov exponent, in order to probe the various regimes described above.

[43] The flow that we choose has two phases: in the first, a plume is compressed in latitude direction and stretched in 


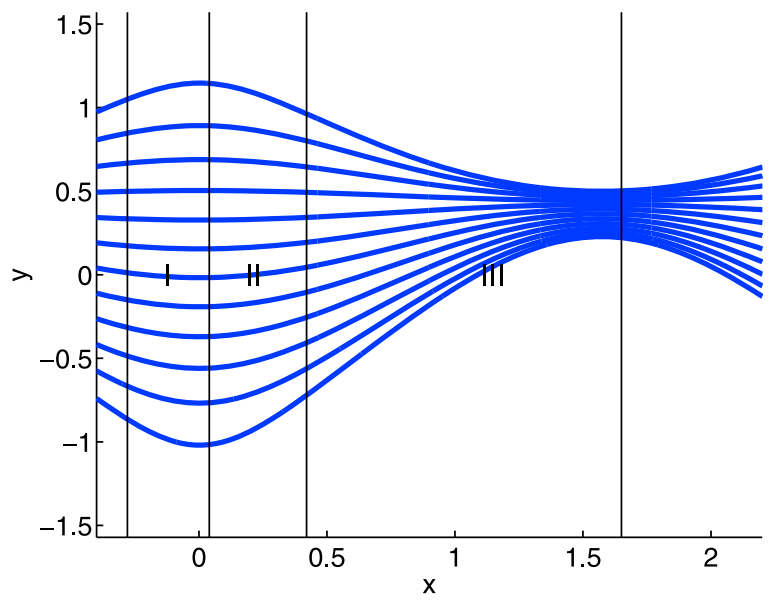

Figure 4. Streamlines of the model convergent-divergent flow introduced in equations (20) and (21), for $\epsilon=5 / 7$ for spherical geometry. A plume entering at the left boundary at $x \approx-0.28$ first expands (region I), then compresses (regions II and III), and then after the first constriction expands again.

longitudinal direction, and in the second it is reexpanded to its original size. The model flow field is as follows:

$$
\begin{gathered}
u=\frac{V}{1+\epsilon \cos (2 x)} \\
v=-\frac{2 V \epsilon\left(y-y_{0}\right) \sin (2 x)}{(1+\epsilon \cos (2 x))^{2} f(y)},
\end{gathered}
$$

with $(u, v)$ the horizontal components, depending on longitude $0 \leq x \leq 2 \pi$ and latitude $-\pi / 2 \leq y \leq \pi / 2$, and

$$
f(y)= \begin{cases}1 & \text { for flat surface geometry } \\ \cos (y) & \text { for spherical surface geometry }\end{cases}
$$

The convergent-divergent flow field is selected to be smooth, incompressible, and periodic in $x$ direction.

[44] The flow is characterized by three parameters: $V$ is the mean westerly wind speed, $\epsilon$ controls the magnitudes of the velocity gradients, and $y_{0}$ measures the location of the stagnation points. We choose $y_{0}=0.43$. The parameters $V, \epsilon$ then control the Lyapunov exponent of the flow through the relation

$$
\lambda \equiv-\nabla_{y}(f(y) v)=\frac{2 V \epsilon \sin (2 x)}{(1+\epsilon \cos (2 x))^{2}}
$$

[45] Figure 4 shows the flow streamlines for $\epsilon=5 / 7$ for spherical geometry. The flow has periodic regions of compression and dilation, with maximum (minimum) compression occurring when $\cos (2 x)=-1(\cos (2 x)=1)$, where the maximum (minimum) longitudinal flow velocity reaches $u_{\max }=V /(1-\epsilon)\left(u_{\min }=V /(1+\epsilon)\right)$. A plume passing through the compression zone in the absence of diffusion is squeezed from an initial width $W$ to a size

$$
W_{0} \approx W u_{\min } / u_{\max }=W(1-\epsilon) /(1+\epsilon) .
$$

[46] Let us summarize our expectations for a plume propagating through this flow. The critical length scale $r_{\mathrm{b}} \sim \sqrt{\Delta x \ell}$, where $\ell$ is the characteristic length scale of the flow. For this flow, (21) and (23) imply that $\ell=v / \lambda=$ $\left(y-y_{0}\right) / \cos (y)$, where $v$ is the meridional velocity component value (21). The characteristic length scale over which the flow varies is set by the plume width itself, so $\ell=W$.

[47] If an underresolved plume propagates in an essentially uniform flow, we expect decay rate $\alpha \sim \Delta x$. In the convergent flow, if the plume width $W>r_{\mathrm{b}}$, then the decay rate scales like $\sqrt{\Delta x}$. If the width value in the convergence zone $W_{0}<r_{\mathrm{b}}$, the plume will broaden so that $W \approx r_{\mathrm{b}}$; the decay rate $\alpha$ then should be independent of $h$.

[48] To test this theory, we consider the propagation of an initial plume with size $12^{\circ} \times 15^{\circ}(1330 \mathrm{~km} \times 1680 \mathrm{~km})$; we consider three different resolutions $4^{\circ} \times 5^{\circ}, 2^{\circ} \times 2.5^{\circ}$ and $1^{\circ} \times 1.25^{\circ}$. These choices imply $r_{\mathrm{b}} \sim 800 \mathrm{~km}, 560 \mathrm{~km}$, $400 \mathrm{~km}$ for each of the three resolutions.

[49] We perform numerical tests for each resolution at two values of $\epsilon$. The values of $\epsilon=5 / 7$ and $\epsilon=0.5$ correspond to the convergence ratio $u_{\max } / u_{\min }=6$ and $u_{\max } / u_{\min }=3$ accordingly. Equation (24) then implies that the width decreases by a factor of 6 and 3 as the plume traverses the compression zone, so that $W_{0}=230 \mathrm{~km}$ for the first case and $460 \mathrm{~km}$ for the second case.

[50] We simulate the advection equation using the Piecewise Parabolic Method. If all spatial scales are well resolved it is possible to achieve a third-order convergence rate, but since the plume boundaries are underresolved the convergence is close to first order in a quasi-uniform flow. Figure 5a shows the maximum concentration $Y_{\max }=\max (Y)$ decay versus time for the three aforementioned resolutions $4^{\circ} \times 5^{\circ}$, $2^{\circ} \times 2.5^{\circ}$ and $1^{\circ} \times 1.25^{\circ}$.

[51] We are interested in the dependence of the decay rate on resolution. The flow moves from near-uniform regime (region I) to a compression regime (region II) to a regime where $W<r_{\mathrm{b}}$ (region III). The average decay rates corresponding to the dashed lines in Figure 5a are plotted in Figure 5b. For $\epsilon=5 / 7$ in region I the decay rate depends roughly linearly on the mesh spacing $\sim \Delta x$; in region II the decay rate depends roughly $\sim \Delta x^{1 / 2}$ and in region III, the decay rate is essentially independent of mesh spacing $\sim \Delta x^{0}$. In region $\mathrm{I}$, the plume size $W \approx 1600 \mathrm{~km}$ is much larger than the largest critical value $r_{\mathrm{b}}=800 \mathrm{~km}$ for the coarsest resolution. In region II, the plume size $W \approx 1000 \mathrm{~km}$ is close to the critical value for the coarsest resolution, causing the error to decrease more slowly. In region III, $r_{\mathrm{b}}=400 \mathrm{~km}$ for the finest grid. This is larger than the compressed width of the plume $W_{0}=230 \mathrm{~km}$, implying that the decay rate becomes independent of the mesh resolution in this region for the considered resolutions. This conclusion is fully confirmed by our calculation (see Figure $5 b$ ).

[52] Figure 5c shows maximum concentration decay $Y_{\max }=\max (Y)$ versus time for the same resolutions $4^{\circ} \times 5^{\circ}$, $2^{\circ} \times 2.5^{\circ}$ and $1^{\circ} \times 1.25^{\circ}$ for the less stretched flow with $\epsilon=0.5$. Note that in Figure 5, the plume stops decaying as soon as the flow stops constricting and begins expanding.

[53] Here the most illustrative plume behavior is observed in the zone of maximum plume stretching. The average decay rates in this region corresponding to dashed lines in Figure $5 \mathrm{c}$ are plotted in Figure 5c. In this zone, the decay rate does not change when the plume resolution increases 

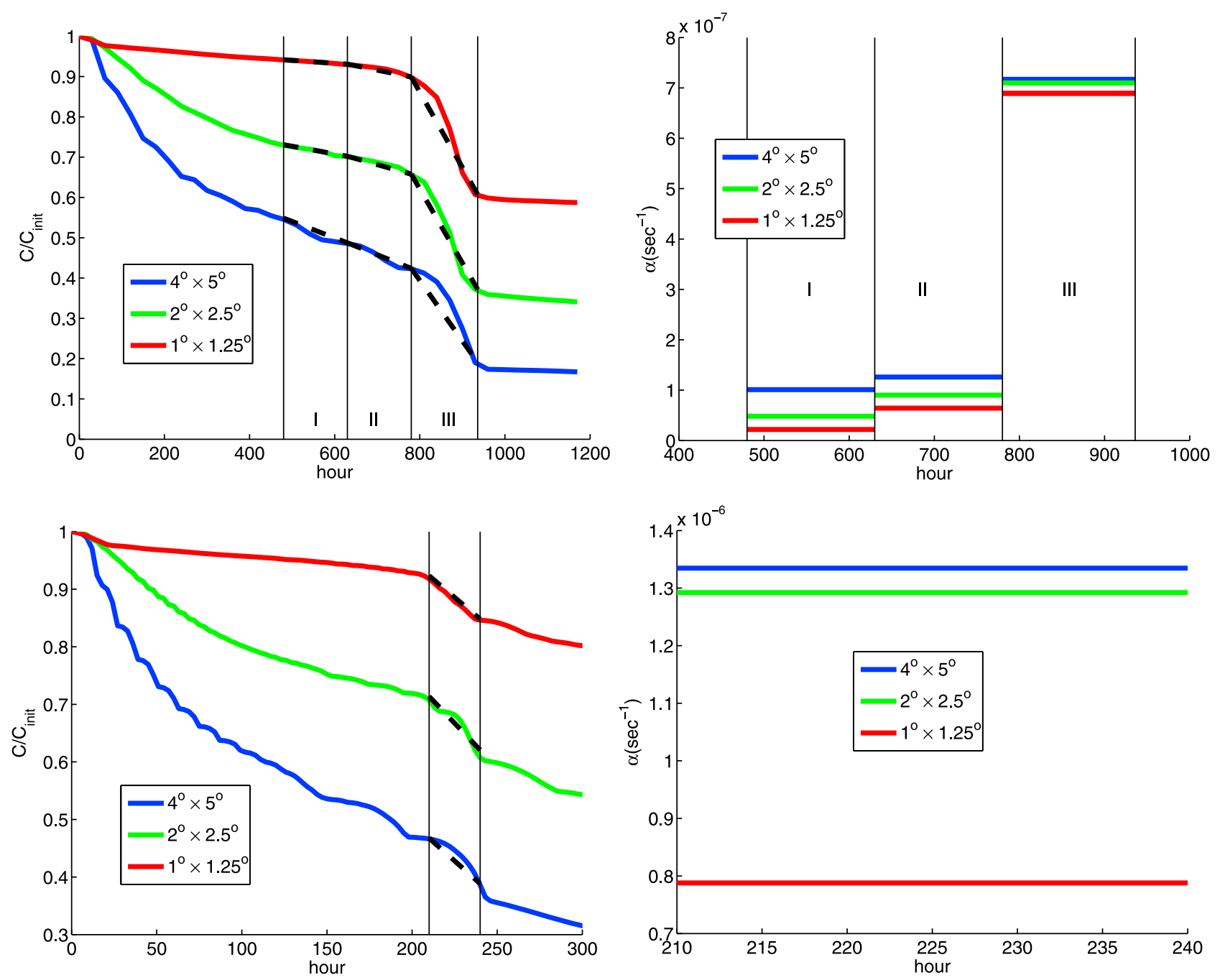

Figure 5. (left) Decay of a plume as a function of time, entering the convergent-divergent flow with velocity field given by equations (20) and (21), and (right) the corresponding average decay rates with ( $a$ and $b) \epsilon=5 / 7$ and $\left(c\right.$ and d) $\epsilon=0.5$. The initial width of the plume is $12^{\circ} \times 15^{\circ}(1330 \mathrm{~km} \times 1680 \mathrm{~km})$. The blue, green, and red lines correspond to resolutions of $4^{\circ} \times 5^{\circ}, 2^{\circ} \times 2.5^{\circ}$, and $1^{\circ} \times 1.25^{\circ}$, respectively. The dashed lines show the average decay rate in the corresponding region. In Figure 5a, regions I, II, III correspond to the decay rate depending roughly $\sim \Delta x, \sim \sqrt{\Delta x}$, and independent of $\Delta x$, respectively. In Figure $5 b$, the decay rate is roughly independent of $\Delta x$ when the resolution increases from $4^{\circ} \times 5^{\circ}$ to $2^{\circ} \times 2.5^{\circ}$, but it reduces with further refinement to $1^{\circ} \times 1.25^{\circ}$.

twice from the crudest resolution $4^{\circ} \times 5^{\circ}$ to the intermediate resolution $2^{\circ} \times 2.5^{\circ}$, which is expected since $W_{0}=460 \mathrm{~km}$ size is below the critical values $r_{\mathrm{b}}$ for both resolutions $(800 \mathrm{~km}$ and $560 \mathrm{~km})$. The numerical decay rate, however, drops $\sim 1.6$ fold when the plume resolution increases twice from the intermediate $2^{\circ} \times 2.5^{\circ}$ to the finest resolution $1^{\circ} \times$ $1.25^{\circ}$. Since $W_{0}=460 \mathrm{~km}$ is close but higher than the critical value $r_{\mathrm{b}}=400 \mathrm{~km}$ the numerical scheme demonstrates slow convergence below the first-order rate. These numerical results agree with our theoretical description: the decay rate does not decrease with the grid refinement if the pollution plume size is below $r_{\mathrm{b}}$. The simulations show that this critical size $r_{\mathrm{b}}$ decreases with spatial resolution and the flow nonuniformity. In compliance with the theory predictions the nonconvergence has been observed for the flow described in the first part of this section when the plume passes through the zone III (refer to Figure 5a) where its size is below the critical value. If the plume size is larger than the critical value, the numerical decay rate decreases with the grid refinement. This regime has been observed for the second case of moderately stretched flow where the plume size is estimated to exceed the critical size while the grid is refined from the resolution $2^{\circ} \times 2.5^{\circ}$ to $1^{\circ} \times 1.25^{\circ}$.

\subsection{Geophysical Flow}

[54] Finally, we examine the efficacy of our theory for pollution plume propagation in a realistic (though 2-D) geophysical flow, using the same implementation of GEOS-Chem used for Figures 1-2. Here we examine the plume dynamics at the same resolutions for the initial $12^{\circ} \times 15^{\circ}(1330 \mathrm{~km} \times 1680 \mathrm{~km})$ plume as in the previous subsection, where the plume begins its trajectory above northeastern China (refer to Figure 2) on 15 June 2004. The maximum concentration versus time for three different 

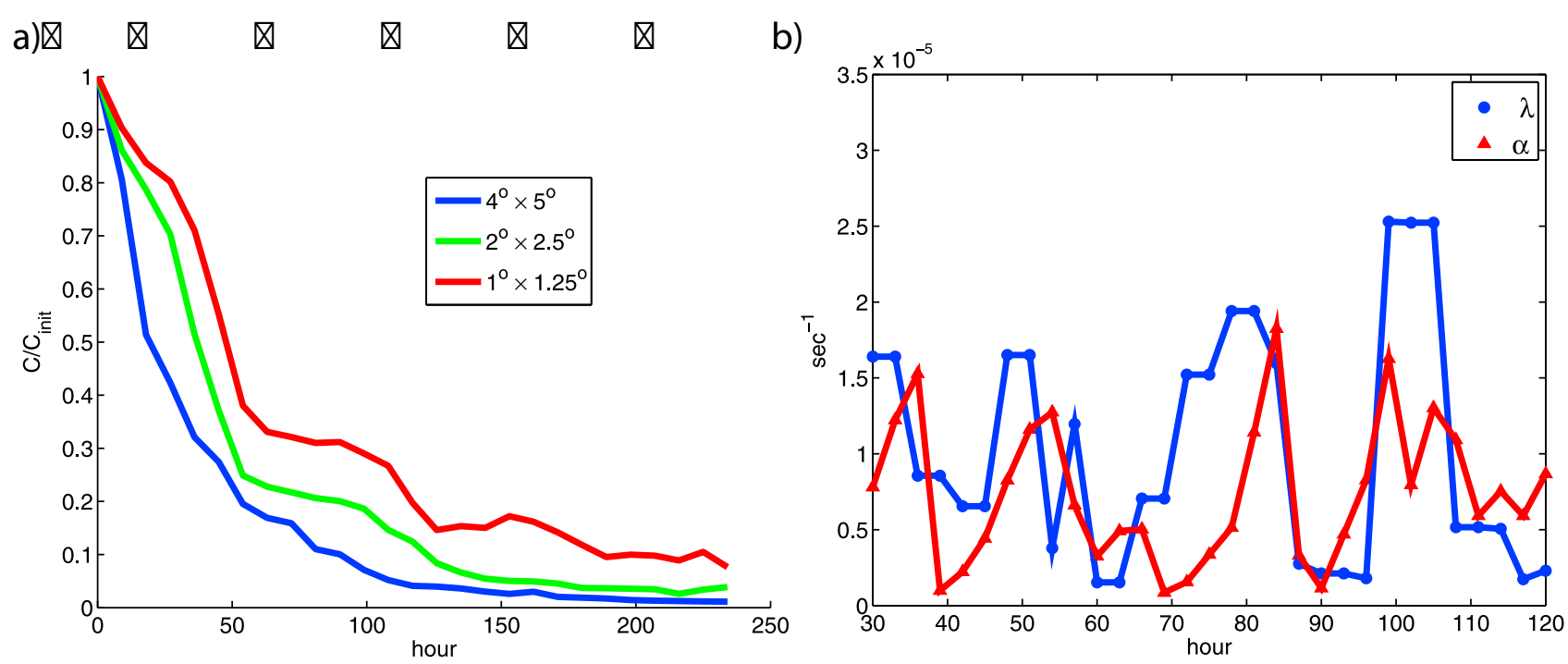

Figure 6. (a) Decay of a pollution plume in a geophysical flow. The initial plume position is identical to that of Figure $2 \mathrm{a}$, though the flow field corresponds to a different initial time. The blue, green, and red curves represent spatial resolutions $4^{\circ} \times 5^{\circ}, 2^{\circ} \times 2.5^{\circ}$, and $1^{\circ} \times 1.25^{\circ}$, respectively. (b) The decay rate of the plume $\alpha \equiv\left|\frac{1}{n} \frac{d n}{d t}\right|$ (red triangles), and the local flow Lyapunov exponent $\lambda$ (blue circle) along the path of the pollution plume for the resolution $4^{\circ} \times 5^{\circ}$. The plume path is defined as the track followed by the maximum concentration of the plume.

resolutions $4^{\circ} \times 5^{\circ}, 2^{\circ} \times 2.5^{\circ}$, and $1^{\circ} \times 1.25^{\circ}$ is presented in Figure 6a. The numerical decay rate drops, albeit slightly, with increasing resolution. Our numerical calculations show that the average characteristic length scale over which the flow varies $\vec{\ell}=\int_{0}^{T} \ell d t / T \sim 2800 \mathrm{~km}$ for the crudest resolution $4^{\circ} \times 5^{\circ}$. This gives us the estimate for the critical plume size $r_{\mathrm{b}} \sim 1000 \mathrm{~km}$ which means that $W \sim r_{\mathrm{b}}$ for the crudest resolution. The slow convergence confirms that the plume size $W$ is in the range of the critical value $r_{\mathrm{b}}$. In order to show this more clearly, we must measure the value of the Lyapunov exponent $\lambda$ along the pollution plume path.

[55] We define the plume path as the path corresponding to the maximum concentration. The most striking demonstration of the effect argued in this paper is shown in Figure 6b, which shows both the Lyapunov exponent $\lambda$ (the velocity gradient along the transport direction) and the decay coefficient $\alpha$ along the plume path. The correlation between $\alpha$ and $\lambda$ is striking; throughout the evolution, $\alpha<\lambda$, suggesting that the propagating plume is in the regime of equation (16), in correspondence with our resolution studies of Figure 6a. The scheme convergence rate however is smaller than $\sqrt{\Delta x}$ predicted by equation (16). This reduction of the scheme convergence is likely caused by increased stretching of the flow for higher resolutions discussed by Wild and Prather [2006]. In order to quantify this effect we consider the dependence of the average value of plume decay rate $\bar{\alpha}=\int_{0}^{T} \alpha d t / T$ and the average flow stretching $\bar{\lambda}=\int_{0}^{T} \lambda d t / T$. Here we average over a time interval $T=240$. Note that although these are time averages, since the plume is moving through the flow field, they are also effectively averaging over the spatial path of the plume. Table 1 presents the values of $\bar{\alpha}$ and $\bar{\lambda}$ for three resolutions considered in the paper. We estimate that $\bar{\lambda} \sim \Delta x^{-0.5}$ for the considered resolutions from the data given in Table 1 . After substituting this expression for $\bar{\lambda}$ into (16) we get $\bar{\alpha} \sim \Delta x^{0.25}$ which is consistent with the $\bar{\alpha}$ dependence from the spatial resolution step given in the Table.

\section{Conclusions}

[56] It this paper we studied the parasitic dilution of synoptic-scale pollution plumes (and more generally any well-defined chemical layer structure) transported by atmospheric chemical transport models (CTMs) on a global scale. The parasitic dilution is caused by numerical mixing which is due to combined effect of numerical diffusion and stretching of the complex and variable geophysical flow. We showed that the effect of plume dilution due to numerical mixing is much stronger in a nonuniform atmospheric flow than in a homogeneous flow. Computational experiments for a plume transported in the free troposphere at midlatitudes at typical resolutions of global CTMs indicate numerical decay rates 40 times faster for a realistic atmospheric flow than for a uniform flow.

[57] We also find that in the regime of global CTMs, the numerical decay rate depends much more slowly on mesh resolution than expected from high order advection schemes. The dependence of the decay rate on mesh spacing $\Delta x$ is defined by the relative size of the plume width $W$ and $r_{\mathrm{b}}=\sqrt{\Delta x \ell}$, where $\ell$ is the characteristic scale of the flow

Table 1. Average Values of Plume Decay Rate $\bar{\alpha}$ and Lyapunov Exponent $\bar{\lambda}$ Along the Pollution Plume Path for Different Resolutions

\begin{tabular}{lcc}
\hline Resolution & $\bar{\alpha} \times 10^{6}\left(\mathrm{~s}^{-1}\right)$ & $\bar{\lambda} \times 10^{6}\left(\mathrm{~s}^{-1}\right)$ \\
\hline $4^{\circ} \times 5^{\circ}$ & 5.5 & 9.0 \\
$2^{\circ} \times 2.5^{\circ}$ & 4.5 & 13.1 \\
$1^{\circ} \times 1.25^{\circ}$ & 3.7 & 18.3 \\
\hline
\end{tabular}


variation. Our findings here are not inconsistent with a recent study comparing the results of two different global CTMs, upon increasing the resolution [Prather et al., 2008].

[58] For a synoptic-scale $\sim 1000-2000 \mathrm{~km}$ plume with $4^{\circ} \times 5^{\circ}$ resolution, the plume width is of order $r_{\mathrm{b}}$, so that the decay rate depends slowly on the mesh spacing, decreasing like $\sqrt{\Delta x}$. This dependence is even weaker if we account for the effect of increased flow stretching for higher resolution. A numerical scheme will recover its normal convergence rate only if $W \gg r_{\mathrm{b}}$. Since the critical plume size $r_{\mathrm{b}}$ also decreases like $\sqrt{\Delta x}$ with grid refinement, a tenfold decrease in $r_{\mathrm{b}}$ requires a hundredfold decrease in the mesh spacing. Hence in order for the atmospheric flow shown in Figure 2 to have a decay rate that is comparable to the uniform flow at the current resolution, we must decrease the mesh spacing in the simulation to a few kilometers. This is unrealistic at a global scale with current computational resources.

[59] More efficient algorithms are needed, examples of which include either spatial reduction algorithms [Rastigejev et al., 2007], which use the spatial separation of the fast reactions to lower computational costs (the algorithm has the potential of reducing computational cost by at least an order of magnitude for a typical atmospheric chemical kinetic mechanism, which would allow finer grids), or adaptive mesh refinement. A final approach is to embed Lagrangian plumes into global atmospheric chemical transport.

\section{Appendix A: Numerical Diffusion Estimates}

[60] Here we discuss the numerical diffusion of the numerical schemes used in global CTMs for plumes whose characteristic size is of order the mesh spacing. Consider the second-order Van Leer method (VLM) [Van Leer, 1974] or for solving the advection equation with constant wind speed $u$

$$
\frac{\partial n}{\partial t}+u \frac{\partial n}{\partial x}=0
$$

The scheme can be written as follows:

$$
\begin{aligned}
n_{0}^{\Delta t} & -n_{0}^{0}=-\sigma \Delta_{-1 / 2} n \\
& -\sigma(1-\sigma)\left(\Delta_{1 / 2} n-\Delta_{-3 / 2} n+S\left(\vartheta_{0}\right)\left(\Delta_{1 / 2} n-\Delta_{-1 / 2} n\right)\right. \\
& \left.-S\left(\vartheta_{-1}\right)\left(\Delta_{1 / 2} n-\Delta_{-3 / 2} n\right)\right) / 4 .
\end{aligned}
$$

The symbols $\Delta_{i-1 / 2}, \vartheta_{i}, S(\vartheta), \sigma$ are defined as follows:

$$
\begin{gathered}
\Delta_{i-1 / 2} n=n_{i}-n_{i-1}, \\
\vartheta_{i}=\frac{\Delta_{i+1 / 2} n}{\Delta_{i-1 / 2} n}, \\
S(\vartheta)=\frac{|\vartheta|-1}{|\vartheta|+1} \\
\sigma=\frac{u \Delta t}{\Delta x}
\end{gathered}
$$

[61] To analyze the scheme, we proceed as discussed in section 3 of the text, and first expand each term of the finite difference equation into Taylor series. The first several terms in the resulting Taylor series expansion are thus as follows:

$$
\begin{aligned}
\frac{\partial n}{\partial t}+u \frac{\partial n}{\partial x}= & \frac{u(1-\sigma)}{4}\left(S\left(\vartheta_{0}\right)-S\left(\vartheta_{1}\right)\right) \Delta x \frac{\partial^{2} n}{\partial x^{2}} \\
& +\frac{u(1-\sigma)}{4}\left(S\left(\vartheta_{0}\right)+\frac{1}{3}\right)(\Delta x)^{2} \frac{\partial^{3} n}{\partial x^{3}}
\end{aligned}
$$

From (A5) the difference $S\left(\vartheta_{0}\right)-S\left(\vartheta_{1}\right)$ depends on the smoothness of the solution; when the $n$ varies smoothly, we have that $S(\vartheta) \sim \Delta x$, so that $S\left(\vartheta_{0}\right)-S\left(\vartheta_{1}\right) \sim \Delta x^{2}$; on the other hand near a sharp boundary the difference between $n$ at neighboring mesh points is order one, so that $S\left(\vartheta_{0}\right)-$ $S\left(\vartheta_{1}\right) \sim 1$. Hence in the vicinity of the plume boundaries the VLM is a first-order diffusive scheme with the value of numerical viscosity $D \sim u(1-\sigma) \Delta x / 4$. Indeed, this result is a general property of advection schemes.

[62] Acknowledgments. We gratefully acknowledge support from the National Science Foundation CMG program for funding the early stages of this research. DJJ acknowledges funding from the NASA Atmospheric Composition Modeling and Analysis Program. MPB acknowledges support from the NSF Division of Mathematical Sciences. YR was partly supported by a grant from the NOAA Educational Partnership Program under cooperative agreement NA06OAR4810187. RP was partly supported by the Korea Meteorological Administration Research and Development Program under grant CATER 2007-3205.

\section{References}

Balkovsky, E., and A. Fouxon (1999), Universal long-time properties of Lagrangian statistics in the Batchelor regime and their application to the passive scalar problem, Phys. Rev. E, 60, 4164.

Bey, I., et al. (2001), Global modeling of tropospheric chemistry with assimilated meteorology: Model description and evaluation, J. Geophys. Res., 106(D19), 23,073.

Chella, R., and J. M. Ottino (1984), Conversion and selectivity modifications due to mixing in unpremixed reactors, Chem. Eng. Sci., 39, 551.

Chertkov, M., and V. Lebedev (2003), Effects on chaotic advectiondiffusion chemical reactions, Phys. Rev. Lett., 90, 134501.

Colella, P., and P. L. Woodward (1984), The Piecewise Parabolic Method (PPM) for gas-dynamical simulations, J. Comp. Phys., 54, 174.

Colette, A., and G. Ancellet (2005), Impact of vertical transport processes on the tropospheric ozone layering above Europe. part ii: Climatological analysis of the past 30 years, Atmos. Environ., 39, 5423

Fairlie, T. D., R. B. Pierce, J. A. Al-Saadi, W. L. Grose, J. M. Russell, M. H. Proffitt, and C. R. Webster (1999), The contribution of mixing in lagrangian photochemical predictions of polar ozone loss over the arctic in summer 1997, J. Geophys. Res., 104(D21), $26,597$.

Fairlie, T. D., M. A. Avery, R. B. Pierce, J. Al-Saadi, J. Dibb, and G. Sachse (2007), Impact of multiscale dynamical processes and mixing on the chemical composition of the upper troposphere and lower stratosphere during the Intercontinental Chemical Transport Experiment-North America, J. Geophys. Res., 112, D16S90, doi:10.1029/2006JD007923.

Hoffman, J. D. (1992), Numerical Methods for Engineers and Scientists, McGraw-Hill, New York.

Hudman, R. C., et al. (2004), Ozone production in transpacific Asian pollution plumes and implications for ozone air quality in California, J. Geophys. Res., 109, D23S10, doi:10.1029/2004JD004974.

Khosrawi, F., J. U. Grooss, R. Muller, P. Konopka, W. Kouker, R. Ruhnke, T. Reddmann, and M. Riese (2005), Intercomparison between Lagrangian and Eulerian simulations of the development of midlatitude streamers as observed by Crista, Atmos. Chem. Phys., 5, 85 .

Legras, B., I. Pisso, G. Berthet, and F. Lefevre (2005), Variability of the lagrangian turbulent diffusion in the lower stratosphere, Atmos. Chem. Phys., 5, 1605.

LeVeque, R. J. (1990), Numerical Methods for Conservation Laws, Birkhauser, Basel, Switzerland.

Lin, S.-J., and R. B. Rood (1996), Multidimensional flux form semiLagrangian transport schemes, Mon. Weather Rev., 124, 2046. 
McKenna, D. S., J. U. Grooss, G. Gunther, P. Konopka, R. Muller, G. Carver, and Y. Sasano (2002), A new chemical lagrangian model of the stratosphere (CLAMS): 2. Formulation of chemistry scheme and initialization, J. Geophys. Res., 107(D15), 4256, doi:10.1029/ 2000JD000113

Newell, R. E., V. Thouret, J. Y. N. Cho, P. Stoller, A. Marenco, and H. G. Smith (1999), Ubiquity of quasi-horizontal layers in the troposphere, Nature, 398, 316.

Pierce, R. B., and T. D. A. Fairlie (1993), Chaotic advection in the stratosphere: Implications for the dispersal of chemically perturbed air from the polar vortex, J. Geophys. Res., 98(D10), 18,589.

Pierce, R. B., J. A. Al-Saadi, T. D. Fairlie, J. R. Olson, R. S. Eckman, W. L. Grose, G. S. Lingenfelser, and J. M. Russell (1999), Large-scale stratospheric ozone photochemistry and transport during the polaris campaign, J. Geophys. Res., 104(D21), 26,525.

Prather, M. J., and A. H. Jaffe (1990), Global impact of the Antarctic ozone hole: Chemical propagation, J. Geophys. Res., 95, 3473, doi:10.1029/ 89JD02940.

Prather, M. J., X. Zhu, S. E. Strahan, S. D. Steenrod, and J. M. Rodriguez (2008), Quantifying errors in trace species transport modeling, Proc. Natl. Acad. Sci. U. S. A., 50, 19,617.

Rastigejev, Y., M. P. Brenner, and D. J. Jacob (2007), Reduction algorithm for atmospheric chemical transport models, Proc. Natl. Acad. Sci. U. S. A., $104,13,875$.

Reames, F. M., and T. H. Zapotocny (1999), Inert trace constituent transport in sigma and hybrid isentropic-sigma models. Part I: Nine advection algorithms, Mon. Weather Rev., 127, 1730.

Seinfeld, J. H., and S. N. Pandis (1998), Atmospheric Chemistry and Physics: From Air Pollution to Climate Change, John Wiley, New York

Staudt, A. C., et al. (2003), Sources and chemistry of nitrogen oxides over the tropical Pacific, J. Geophys. Res., 108(D2), 8239, doi:10.1029/ 2002JD002139.

Stohl, A., O. R. Cooper, and P. James (2004), A cautionary note on the use of meteorological analysis fields for quantifying atmospheric mixing, J. Atmos. Sci., 61(12), 1446.
Thouret, V., et al. (2000), General characteristics of tropospheric trace constituent layers observed in the MOZAIC program, J. Geophys. Res., $105,17,379$

Van Leer, B. (1974), Toward the ultimate conservative difference scheme. II. Monotonicity and conservation combined in a second-order scheme, J. Comp. Phys., 14, 361

Vuolo, M. R., H. Chepfer, L. Menut, and G. Cesana (2009), Comparison of mineral dust layers vertical structures modeled with CHIMERE-DUST and observed with the CALIOP lidar, J. Geophys. Res., 114, D09214, doi:10.1029/2008JD011219.

Waugh, D. W. (1993), Subtropical stratospheric mixing linked to disturbances on the polar vortices, Nature, 365, 535 .

Weaver, C. J., A. R. Douglass, and R. B. Rood (2000), Lamination frequencies as a diagnostic for horizontal mixing in a $3 \mathrm{~d}$ transport model, J. Atmos. Sci., 57(2), 247.

Wild, O., and M. J. Prather (2006), Global tropospheric ozone modeling: Quantifying errors due to grid resolution, J. Geophys. Res., 111, D11305, doi:10.1029/2005JD006605.

Winker, D. M., W. H. Hunt, and M. J. McGill (2007), Initial performance assessment of CALIOP, Geophys. Res. Lett., 34, L19803, doi:10.1029/ 2007GL030135.

M. P. Brenner and D. J. Jacob, School of Engineering and Applied Sciences, Harvard University, Cambridge, MA 02138, USA. (brenner@ seas.harvard.edu)

R. Park, School of Earth and Environmental Sciences, Seoul National University, 599 Gwanangno, Gwanak-gu, Seoul, 151-742, South Korea.

Y. Rastigejev, Department of Mathematics, North Carolina Agricultural and Technological State University, 1601 East Market St., Greensboro, NC 27411, USA. 